\title{
The importance of understanding the molecular mechanism of stem cell-induced cardiac tissue
} repair

\begin{abstract}
“... a patient is predisposed to stem cell-based healing as either a 'healer' or a 'scarrer'. If they are a healer, they recover from end-organ injury but enhance dysplastic tissue/tumor growth, whereas if they are a scarrer, they scar in response to tissue injury and are prone to atherosclerosis and ischemic events but do not support tumor growth."
\end{abstract}

\section{KEYWORDS: acute myocardial infarction $=$ chemokines " chronic heart failure w endogenous stem cell repair $\approx$ stem cells}

The potential for stem cell repair of injured tissue has garnered great hope for the prevention and treatment of cardiac dysfunction. Early studies suggested that the direct transplantation of bone marrow-derived stem cells resulted in improved cardiac function in acute myocardial infarction (AMI) and chronic heart failure through the direct regeneration of lost cardiac myocytes $[1,2]$. In the early 2000 s, there was significant controversy regarding the validity of cardiac differentiation of stem cells following transplantation and engraftment in acutely injured myocardium [3]. At that time, lost in the controversy regarding cardiac myocyte regeneration was the fact that stem cell transplantation in AMI and chronic heart failure led to improvements in cardiac function and remodeling $[1,4-7]$.

Over the ensuing several years there was increasing understanding of the importance of the paracrine effects of stem cell-based tissue repair. Work from Dzau and colleagues nicely demonstrated that conditioned media from mesenchymal stem cells (MSCs) could induce the same benefits as engraftment of the MSCs themselves and that the effects of conditioned media could be modulated and enhanced by manipulation of the MSC secretome [8]. Mayorga and colleagues further refined the signaling pathways responsible for cardiac protein expression in MSCs, ultimately demonstrating that cardiac protein expression in MSCs in response to TGF $\beta$ was not associated with cardiac regeneration but actually enhancement of the MSC secretome $[9,10]$.

We participated in developing the multipotent adult progenitor cell (MAPC) [11] to prevent cardiac dysfunction in AMI. In our preclinical studies we demonstrated significant preservation of cardiac function and improvement in neovascularization in the post-AMI heart despite the limited survival of the MAPC beyond 6 weeks after AMI [12,13]. These data further supported that the benefits of adult stem cell therapy were not linked to cardiac regeneration but rather paracrine factor release and enhancement of endogenous tissue repair. The fact that nonsurviving stem cells could induce a clinical benefit was demonstrated in the first-inman MAPC study in which MAPCs were delivered to the adventitia of patients 2-5 days after primary stenting for first AMI [14]. In this study we demonstrated a significant dose-dependent increase in ejection fraction at 4 months after AMI that was sustained for 1 year despite the lack of MAPC survival.

Against the backdrop of the field's focus on the need for stem cells as a therapeutic agent, in 2000 we proposed the following hypothesis: stem cell-based repair of injured tissue is a natural process that, owing to dysregulation of the reparative system rather than lack of stem cells, does not lead to significant clinical benefit. At that time we sought to define the molecular regulators of endogenous stem cell repair of injured tissue. The impact of individual factors on benefits observed through the delivery of stem cell therapy has begun to be defined through the following two studies. We initially identified SDF-1 as a key regulator of stem cell recruitment to the heart following AMI [15] and demonstrated that the overexpression of SDF-1 first through cell-based gene transfer and later standalone gene transfer in ischemic cardiomyopathy led to improvements in cardiac dimensions and function [15-18]. In subsequent studies

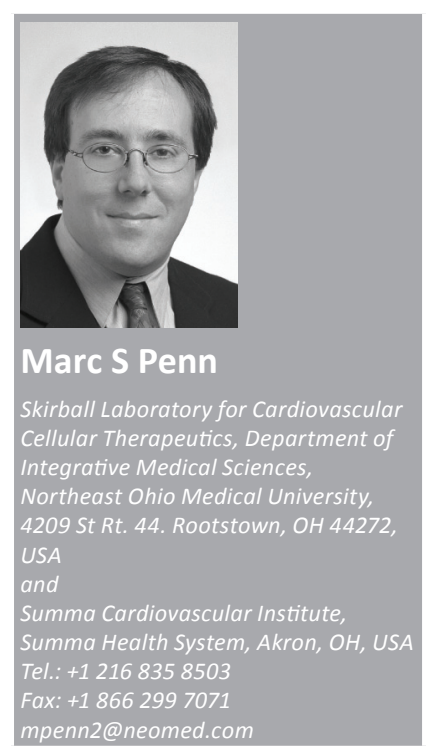

\section{(6) Regenerative Medicine}


we identified a critical role for MCP-3 as a MSC homing factor [19], as well as roles for disabled-2 and NOTCH signaling in endogenous stem cell repair $[9,10]$. Recently, using mice that lack cardiac myocyte and cardiac stem cell CXCR4 expression, we demonstrated that the release of SDF-1 by MSCs is critical for MSC-induced inhibition of cardiac myocyte death and enhancement of cardiac stem cell recruitment [20]. These data demonstrate that an important mechanism of the effect of adult stem cell therapy is activation of endogenous stem cell repair and support our prior hypothesis that one benefit of adult stem cell therapy is the temporal realignment of SDF-1 secretion with CXCR4 receptor expression by end-organ cells (cardiac myocytes) and endogenous tissue-specific stem cells (cardiac stem cells) [21]. The importance of temporal alignment of the SDF-1-CXCR4 axis has also been shown in the eye in response to retinal detachment $[22,23]$.

\section{"...one benefit of adult stem cell therapy is the temporal realignment of SDF-1 secretion with CXCR4 receptor expression by end-organ cells..."}

In 2011 we began to test the hypothesis that defining the molecular mechanisms of stem cellbased tissue repair could lead to novel theratics in a Phase I clinical trial that sought to reestablish SDF-1 expression in the myocardium of patients with New York Heart Association (NYHA) Functional Classification class III chronic heart failure due to a history of myocardial infarction (NCT01082094). In this study we delivered 5, 15 or $30 \mathrm{mg}$ of JVS-100, a DNA plasmid encoding human SDF-1 [18]. In this invasive first-in-man study, a placebo control was not an option but the $5 \mathrm{mg}$ dose was anticipated to be nontherapeutic. In this recently published study we observed [24]:

- Overexpression of SDF-1 for approximately 17 days [17] in the heart is safe;

- Resolution of peri-infarct ischemia in the 15 and $30 \mathrm{mg}$ cohorts at 4 months;

- Clinically meaningful improvements in 6 min walk, quality of life and NYHA classification at 4 months, sustained to 12 months especially in the $30 \mathrm{mg}$ cohort;

- Stabilization of cardiac function in the 15 and 30 mg cohorts;

- A decline in the clinical and functional status at 12 months in the $5 \mathrm{mg}$ cohort.
As a small (17 patient) Phase I study these data should be considered as proof of concept at best. Two double-blind, randomized, placebocontrolled studies measuring the effects of SDF-1 overexpression in patients with chronic heart failure (NCT01643590) and critical limb ischemia (NCT01410331) are ongoing and will further validate our hypothesis.

It is well recognized that one of the advantages of stem cell therapies is that multiple indications can be treated with the same preparation. Based on this observation, arguably one feature of defining a relevant mechanism of stem cellbased repair is that the mechanism is active in multiple disease or injury states. Consistent with this, we have recently demonstrated that inducing SDF-1 overexpression improves surgical wound healing leading to faster wound closure with significantly less scarring [25]. These observations are now being tested clinically in a study of patients undergoing median sternotomy for open-heart surgery (NCT01657045).

Stem cell-based therapies have tremendous potential to prevent and treat the morbidity and mortality associated with chronic disease and end-organ dysfunction. Clinical trials testing the safety and effects of stem cells to prevent and treat cardiac dysfunction have overwhelmingly demonstrated the safety of the approach. Some trials have demonstrated meaningful and potentially clinically important findings [26,27], whereas others have failed to demonstrate benefits [28-30]. It is clear that adult stem cells trials will move forward testing the multiple different approaches that have developed the cardiovascular regenerative medicine space [31]. The overwhelming data suggest that these adult stem cells lead to activation of an endogenous stem cell-based repair system; thus, the question is will future approaches utilize cells at all? In medicine when we want to induce the immune system we do not transfuse B and T cells, we deliver adjuvants. The data to date suggest that there are adjuvants that prime endogenous stem cell repair. Whether the target is SDF-1 or other chemokines/growth factors remains to be determined. Importantly, the field, funding agencies and peer-review system need to move from hand-waving away the issues of mechanism of action and move to rigorous hypothesis-based science that defines the biology of endogenous stem cell repair.

Perhaps just as important as developing therapies that can lead to improved clinical outcomes is the potential to greatly expand our understanding of pathophysiology more broadly. A 
decade ago when we proposed stem cell-based repair was a natural but clinically inefficient process, a corollary to our hypothesis was that mammals have evolved to scar instead of heal in an attempt to minimize tumor. While still an unproven hypothesis, we would be remiss if we did not note that upregulation of SDF-1, MCP-3 and NOTCH, and downregulation of disabled-2 - all factors that in our hands enhance stem cell function - are all associated with the enhancement of tumor survival. These observations have led us to propose the concept that a patient is predisposed to stem cell-based healing as either a 'healer' or a 'scarrer'. If they are a healer, they recover from end-organ injury but enhance dysplastic tissue/tumor growth, whereas if they are a scarrer, they scar in response to tissue injury and are prone to atherosclerosis and ischemic events but do not support tumor growth. While an unproven hypothesis at this time, it is testable. Through defining the molecular mechanisms associated with stem cell-based tissue repair these types of interesting and transformative hypotheses can be generated.

\section{Financial \& competing interests disclosure}

This work was funded by the generous support of the Skirball Foundation and the Corbin Foundation. MS Penn is named as an inventor on patents for the use of SDF-1 for the prevention and treatment of heart cardiac dysfunction and critical limb ischemia. He is the founder and chief medical officer of Juventas Therapeutics, Inc. and SironRX Therapeutics, Inc., for which he receives equity, consulting fees and royalties. MS Penn receives research funding from Athersys, Inc. and in the last 12 months has honoraria for serving on the scientific advisory board of Aastrom Biosciences, Inc. and the data safety monitoring board for BIOMET, Inc. MS Penn also received honoraria from Longevity Therapeutics, Inc. for serving on their Scientific Advisory Board. The author has no other relevant affiliations or financial involvement with any organization or entity with a financial interest in or financial conflict with the subject matter or materials discussed in the manuscript apart from those disclosed.

No writing assistance was utilized in the production of this manuscript.

\section{References}

1 Orlic D, Kajstura J, Chimenti S et al. Bone marrow cells regenerate infarcted myocardium. Nature 410(6829), 701-705 (2001).

2 Orlic D, Kajstura J, Chimenti S et al. Mobilized bone marrow cells repair the infarcted heart, improving function and survival. Proc. Natl Acad. Sci. USA 98(18), 10344-10349 (2001).

3 Murry CE, Soonpaa MH, Reinecke $\mathrm{H}$ et al. Haematopoietic stem cells do not transdifferentiate into cardiac myocytes in myocardial infarcts. Nature 428(6983), 664-668 (2004).

4 Kocher AA, Schuster MD, Szabolcs MJ et al. Neovascularization of ischemic myocardium by human bone-marrow-derived angioblasts prevents cardiomyocyte apoptosis, reduces remodeling and improves cardiac function. Nat. Med. 7(4), 430-436 (2001).

5 Zhang D, Fan GC, Zhou X et al. Overexpression of CXCR4 on mesenchymal stem cells augments myoangiogenesis in the infarcted myocardium. J. Mol. Cell Cardiol. 44(2), 281-292 (2008).

6 Assmus B, Schachinger V, Teupe C et al. Transplantation of progenitor cells and regeneration enhancement in acute myocardial infarction (TOPCARE-AMI). Circulation 106(24), 3009-3017 (2002).

7 Assmus B, Fischer-Rasokat U, Honold J et al. Transcoronary transplantation of functionally competent BMCs is associated with a decrease in natriuretic peptide serum levels and improved survival of patients with chronic postinfarction heart failure: results of the TOPCARE-CHD Registry. Circ. Res. 100(8), 1234-1241 (2007).

8 Gnecchi M, He H, Liang OD et al. Paracrine action accounts for marked protection of ischemic heart by Akt-modified mesenchymal stem cells. Nat. Med. 11(4), 367-368 (2005).

9 Mayorga ME, Dong F, Sundararaman S et al. Central role for disabled-2 in mesenchymal stem cell cardiac protein expression and functional consequences after engraftment in acute myocardial infarction. Stem Cells Dev. 20, 681-693 (2011).

10 Mayorga ME, Penn MS. miR-145 is differentially regulated by TGF $\beta 1$ and ischemia and targets disabled-2 expression and wnt $/ \beta$-catenin activity. J. Cell Mol. Med. 16(5), 1106-1113 (2012).

11 Schwartz RE, Reyes M, Koodie L et al. Multipotent adult progenitor cells from bone marrow differentiate into functional hepatocyte-like cells. J. Clin. Invest. 109(10), 1291-1302 (2002).

12 Van't HW, Mal N, Huang Y et al. Direct delivery of syngeneic and allogeneic largescale expanded multipotent adult progenitor cells improves cardiac function after myocardial infarct. Cytotherapy 9(5), 477-487 (2007).

13 Medicetty S, Wiktor D, Lehman N et al. Percutaneous adventitial delivery of allogeneic bone marrow derived stem cells via infarct related artery improves long-term ventricular function in acute myocardial infarction. Cell Transplant. 21(6), 1109-1120 (2012).

14 Penn MS, Ellis S, Gandhi S et al. Adventitial delivery of an allogeneic bone marrow-derived adherent stem cell in acute myocardial infarction: Phase I clinical study. Circ. Res. 110(2), 304-311 (2012).

15 Askari A, Unzek S, Popovic ZB et al. Effect of stromal-cell-derived factor-1 on stem cell homing and tissue regeneration in ischemic cardiomyopathy. Lancet 362, 697-703 (2003).

16 Deglurkar I, Mal N, Mills WR et al. Mechanical and electrical effects of cell-based gene therapy for ischemic cardiomyopathy are independent. Hum. Gene Ther. 17(11), 1144-1151 (2006).

17 Sundararaman S, Miller TJ, Pastore JM, Kiedrowski M, Aras R, Penn MS. Plasmidbased transient human stromal cell-derived factor-1 gene transfer improves cardiac function in chronic heart failure. Gene Ther. 18(9), 867-873 (2011).

18 Penn MS, Pastore J, Miller T, Aras R. SDF-1 in myocardial repair. Gene Ther. 19, 583-587 (2012).

19 Schenk S, Mal N, Finan A et al. Monocyte chemotactic protein-3 is a myocardial mesenchymal stem cell homing factor. Stem Cells 25(1), 245-251 (2007).

20 Dong F, Harvey J, Finan A, Weber K, Agarwal U, Penn MS. Myocardial CXCR4 
expression is required for mesenchymal stem cell mediated repair following acute myocardial infarction. Circulation 126(3), 314-324 (2012).

21 Penn MS. Importance of the SDF-1:CXCR4 axis in myocardial repair. Circ. Res. 104(10), 1133-1135 (2009).

22 Penn MS. SDF-1:CXCR4 axis is fundamental for tissue preservation and repair. Am. J. Pathol. 177(5), 2166-2168 (2010).

23 Otsuka H, Arimura N, Sonoda S et al. Stromal cell derived factor- 1 is essential for photoreceptor cell protection in retinal detachment. Am. J. Pathol. 177, 2268-2277 (2010).

24 Penn MS, Mendelsohn FO, Schaer GL et al. An open-label dose escalation study to evaluate the safety of administration of nonviral stromal cell-derived factor-1 plasmid to treat symptomatic ischemic heart failure. Circ. Res. 112(5), 816-825 (2013).

25 Rabbany SY, Pastore J, Yamamoto M et al. Continuous delivery of stromal cell-derived factor-1 from alginate scaffolds accelerates wound healing. Cell Transplant. 19(4), 399-408 (2010).

26 Penn MS, Ellis S, Gandhi S et al. Adventitial delivery of an allogeneic bone marrow-derived adherent stem cell in acute myocardial infarction: Phase I clinical study. Circ. Res. 110(2), 304-311 (2012).

27 Schachinger V, Erbs S, Elsasser A et al. Intracoronary bone marrow-derived progenitor cells in acute myocardial infarction. N. Engl. J. Med. 355(12), 1210-1221 (2006).

28 Traverse JH, Henry TD, Ellis SG et al. Effect of intracoronary delivery of autologous bone marrow mononuclear cells 2 to 3 weeks following acute myocardial infarction on left ventricular function: the LateTIME randomized trial. JAMA 306(19), 2110-2119 (2011).

29 Perin EC, Willerson JT, Pepine CJ et al. Effect of transendocardial delivery of autologous bone marrow mononuclear cells on functional capacity, left ventricular function, and perfusion in chronic heart failure: the FOCUS-CCTRN trial. JAMA 307(16), 1717-1726 (2012).

30 Traverse JH, Henry TD, Pepine CJ et al. Effect of the use and timing of bone marrow mononuclear cell delivery on left ventricular function after acute myocardial infarction: the TIME randomized trial. JAMA 308(22), 2380-2389 (2012).

31 Penn MS, Dong F, Klein S, Mayorga ME. Stem cells for myocardial regeneration. Clin. Pharmacol. Ther. 90(4), 499-501 (2011). 